\title{
Nach hundert Jahren.
}

Vor Wilhelm Windelband.

Wenn man auf die Bewegungen der Philosophie in den letzten Jahrzehnten zurückblickt und in der Gegenwart Umschau hïlt, so ist man wohl versucht, sich staunend zu fragen, ob denn wirklich schon ein Jahrhundert dahingegangen ist, seit der grosse Denker in Königsberg die müden Augen schloss: so unmittelbar lebendig sind uns seine Probleme und Begriffe, so unablässig arbeiten wir noch heute an der Ausspinnung der Gedanken, die er angelegt hat. Und diese historische Macht der kritischen Philosophie zeigt sich gerade darin, dass sie nicht etwa in der Gestalt eines geschlossenen Schulverbandes weiter gewirkt hat, sondern die ganze Breite des wissenschaftlichen Lebens befruchtend durchdrungen hat. Zahlreiche eindrucksvolle und gedankenmächtige Systeme der Philosophie sind in der direkten Weiterentwickelung aus dem Kritizismus erwachsen; aber keines von ihnen hat dauernd die Allgemeinheit und Tiefe der Wirkung auszuüben vermocht wie das Kantische. Dabei erleben wir den eigenartigen Vorgang - ein leuchtendes Beispiel von den sachlichen Notwendigkeiten, die in der Geschichte der Philosophie walten -, dass, nachdem Kants Lehre ihren zweiten Siegeszug gehalten hat, abermals aus ihr kräftige Triebe hervorzubrechen beginnen, die jenen der ersten Entwickelung verwandt und ähnlich sind.

So stehen wir heute, nach hundert Jahren, wiederum vor der Frage: was soll aus dem Kritizismus werden? Fin unvergleichlicher Reichtum von bedeutsamen Prinzipien ist in Kants Denken vereinigt, ohne seine völlige und entschiedene Ausgleichung gefunden $\mathrm{zu}$ haben: je energischer sich die neue Entwickelung darin vertieft hat, um so unabweisbarer ist auch für sie die Forderung geworden, $z \mathfrak{u}$ dem Ganzen neu Stellung $z \mathfrak{u}$ nehmen. Wiederum stehen wir vor der Frage: wie müssen wir Kant recht verstehen, um über ihn hinauszugehen? 
Der Durchbruch dieser Einsicht, den wir gegenwärtig feststellen können, hängt mit allgemeiuen Wandlungen des wissenschaftlichen Geistes zusammen, die sich auch in dem Wechsel der Auffassung des Kritizismus gespiegelt haben. Der neue Aufschwung der Kantischen Lehre, der um das Jahr 1860 herum begann, fiel in die Zeit des Tiefstands von philosophischem Interesse, der fast leidenschaftlichen Ablehnung metaphysischer Fragen und der Beschränkung auf die Arbeiten der Spezialwissenschaften. Aus dieser Stimmung heraus ergriff man begierig, namentlich von Seiten der Naturforscher, eine philosophische Lehre, welche die Unerkennbarkeit der Dinge-an-sich festzulegen und zugleich das Recht einer mathematischen Theorie der Erfahrungswelt zu begründen versprach. So wirkte Kant zunächst, gerade wie bei seinen Lebzeiten, wieder mit den negativen Ergebnissen seiner Erkenntnislehre. Damals war es eine Kontrastwirkung gewesen gegen die Alleswisserei des Rationalismus und des Popularphilosophentums, die der Alles Zermalmende ein für alle Mal abthat: jetzt war es eine Erscheinung der Sympathie, mit der man sich an der philosophischen Rechtfertigung des eigenen Empirismus freuen zu dürfen glaubte. Dieser Sachlage entsprach es, dass die ersten Auffassungen und Umwandlungen, die der "Neukantianismus" erfuhr, den "Antimetaphysizismus" besonders betonten und sich selbst z. T. relativistischen und positivistischen Neigungen zuwandten.

Allein diese "agnostische“ Stimmung hielt nicht Stand. Von Jahrzehnt zu Jahrzehnt wuchs auch in den besonderen Wissenschaften wieder das Bewusstsein von der Aufgabe und dem Bedürfnis, die Fülle des thatsächlichen Materials und die Formen seines wissenschaftlichen Zusammenhanges in letzter Instanz unter allgemeinere Prinzipien zu ordnen, und unter der Einwirkung so bedeutsamer Prinzipien wie der Erhaltung der Energie oder der Entwickelung mehrten sich die Versuche, das Ganze der gewonnenen Einsichten wieder in grossen Linien zusammenzuschauen. Je kräftiger dabei die erneuerte Erkenntnistheorie des Kritizismus behilflich gewesen war, den Materialismus zu überwinden, der halb bewusst, halb unbewusst die naive Grundmeinung während jener Ebbe des philosophischen Denkens ausgemacht hatte, umsomehr erstarkten im Gegensatz dazu alle Denkmotive, welche auf die Erfassung eines geistigen Lebensgrundes der Dinge, im Gedanken, in der Phantasie, im Willen gerichtet sind. Mit diesen Wand- 
lungen aber vereinigten sich heftigere Strönungen in weiteren Kreisen. Durch gewaltige Geschicke und mächtige Umwälzungen des öffentlichen Lebens im Tiefsten aufgeregt, von fieberhaftem Bedürfnis nach neuer Selbstgestaltung ergriffen, verlangte die Volksseele nach dem bestimmten und bestimmenden Ausdruck dessen, was sie bewegt: in Kunst und Litteratur hastet und tastet sie nach dem Ungewöhnlichsten, un sich daran und darin zu formen; und in der Bedrängnis ihrer socialen und religiösen Erregungen erheischt sie gebieterisch von der Philosophie das, ohne das noch keine Zeit zu schöpferischer Gestaltung gelangt ist: eine Weltanschaumng. So haben wir es erfahren, wie am Ende des neunzehnten Jahrhunderts Wissenschaft und Leben deu „Mut der Wahrheit" wiedergefunden haben, den Hegel an seinem Anfange verlangt und den es verloren hatte.

Diese Entwickelung bat der "Neukantianismus" mitgemacht: ihr gemäss sind in der Auffassung und Darstellung, wie in der selbständigen Weiterbildung der Kantischen Lehre Schritt für Schritt mehr die positiven Elemente zur Geltung gekommen, und das allgemeine Interesse am Kritizisinus geht heute, wie vor hundert Jahren, wieder auf die Frage, ob er uns in seinem Grundriss und in seiner Ausführung als philosophische Weltanschauung genügen kann, ob er die Tragkraft und die Erweitèrungsfähigkeit besitzt, um den Reichtum des neuen Lebeus in sich aufzunehmen und sich einzugliedern.

Dass diese positiven Momente und ihre Zusanmenfassung $\mathrm{zu}$ einer Weltanschauung bei Kant vorhanden sind, steht ausser Frage. Die geschichtliche Wucht seiner Erscheinung wäre ohne dies unbegreiflich. Und seine ersten grossen "metaphysischen" Nachfolger, die ganze Generation von Fichte bis zu Herbart und Schopenhauer, haben diese Momente sich nicht entgehen lassen: sie haben daraus die Bausteine gemacht, mit denen sie die kühnen Gebäude ihrer metaphysischen Systeme aufrichteten; jedes darunter hat seine Grundlage in der Weltanschauung des "kritischen" Philosophen.

Darum ist der Streit, ob Kant ein Metaphysiker war, ein Wortstreit gewesen. Es ist offenkundig, dass Kant das, was er Wissenschaft nannte, mit zwingenden Gründen als unfähig zur Überschreitung der Grenzen der Erfahrung, zur Erkenntnis der Dinge-an-sich, zum Aufbau einer Metaphysik im Sinne der „Wissenschaft vom Übersinnlichen" erwiesen hat. Aber es ist ebenso 
offenkundig, dass er von der Realităt der "intelligiblen Welt" unerschiitterlich iiberzeugt war, und dass er von ihrem Iuhalt und Leben, wie von ihren Beziehnngen zur Erscheinungswelt sehr bestimmte und wohldurchdachte Vorstellungen hatte. Der ganze Bestand seiuer philosophischeu Lebeusarbeit enthält eine streng geschlossene und völlig ausgebildete Weltanschauung: und sie liegt nicht etwa nur keimartig zu Grunde oder andeutungsweise im Hintergrunde, sondern offen ausgesprochen zu Tage. Wer das gewaltigste seiner Werke, die Kritik der Urteilskraft, begriffen hat, krmn darïber nicht im Zweifel sein, ebensowenig aber auch über die Bedentung, die der Philosoph dafiir in Anspruch nimmt. Diese Weltanschaunng gilt ihm nicht als eine bloss persönliche Meinung, sie ist nicht seine Privatmetaphysik, die ebenso wie vielleicht andere neben der Erfahrungswissenschaft nur so herlaufen wollte oder dürfte, - sondern er verlangt für sie die „notwendige und allgemeine Geltung" in nicht geringerem Masse als für die mathematisch-naturwissenschaftliche Erkenntnis der Erscheinungen. Die Postulate der praktischen Vernunft beziehen sich auf ihre intelligiblen "Gegenstände" gerade so notwendig wie die Anschaunngen und die Kategorien anf die Sinnenwelt, und die heuristischen Prinzipien der teleologischen Urteilskraft erfassen das Ganze der Natur und des Lebens gerade so allgemeingiltig, wie die „Grundsätze“ auf die Erfahrung angewendet werden. Die Aufdeckung ihrer transscendentalen Geltung gehört zu den Aufgaben der kritischen Philosophie mindestens in demselben Grade wie die Untersuchung über die Bedingungen der Erfahrung. Was man in den Anfängen des Neukantianismus vielfach wach Schopenhauerschem Rezept als Beiwerk angesehen hat, erweist sich als integrierender, vielleicht als der inhaltlich bedeutsamere Teil der kritischen Philosophie, die eben deshalb der systematischen Gliederung und des architektonischen Aufbaues, den ihr Kant gegeben hat und geben musste, auch in ihrer weiteren Entwickelıng nicht entraten kann. Nur so bleibt die Einheit und die gegenseitige Ergänzung der negativen und der positiven Ergebnisse gewahrt, welche das eigenartige Wesen des Kritizisnus ausmacht.

Denn eben darin besteht dessen Grösse und Originalität, dass Kant uns gelehrt hat, Grïnde und Inhalte der Weltanschanung, welche die Philosophie bieten soll, nicht bloss in der wisseuschaftlichen Theorie, sondern im gesamten Umfange des Vernunftlebens zu suchen. Der Einschlag, den früher die „Metaphysiker" 
aus naiven Antrieben des ethischen, ästhetischen oder religiösen Bewusstseins in das wissenschaftliche Begriffsgewebe eingewirkt haben, um ihre philosophische Gesamtanschauung zu gestalten, wird von Kant mit vollem kritischen Bewusstsein in seiner Unentbehrlichkeit erkannt, in seiner Begründung gerechtfertigt, in seinen Auforderungen geregelt: eben damit aber wird das Mass der Ansprïche beschränki, welche die wissenschaftliche Theorie für sich allein in Rahmen der philosophischen Weltanschauung zu erheben befugt ist. Das ist im letzten Grunde das Wesen der Epoche, welche Kant in der Geschichte des menschlichen Denkens gemacht hat: und darin besteht die aktuelle Bedeutung seiner Lehre für ein Zeitalter, das, wie das gegenwärtige, wieder einmal die Urrechte der Gefühle und der Triebe in der Gestaltung seines gesamten Lebens und damit auch seincr intellektuellen Überzeugungen anerkannt sehen möchte.

Diese Bedeutung steht um so höher, als fïr den leidenschaftlichen Überschwang und dic naturalistische Ungezügeltheit solcher Bestrebungen, wie sie sich ja sattsam in der pepularphilosophischen Litteratur unserer 'lage breit machen, kein besseres Heilınittel ist als die kritische Philosophie selber. Demn die Bedeutsamkeit ethischer und ästhetischer Bedürfnisse für die philosophische Weltanschauung erkennt Kant nun und nimmermehr in ihrer unmittelbaren, eiuzelnen, historisch bedingten individuellen Erscheinungsweise, sondern nur in der Gestalt an, welche sie für die Vernunft, d. h. in allgemeingiltiger und notwendiger Weise besitzen. Die Elemente der "Metaphysik“, wie er sie verlangt, sind sehr verschieden, abor gemeinsam ist ihnen allen die notwendige und allgemeine Geltung für die „Vernunft“, für das „Bewusstsein uiberhaupt". Allein eben deshalb sind in der kritischen Philosophie die Gründe der Allgemeingiltigkeit und Notwendigkeit in den verschiedenen Bereichen des Wirklichen verschieden: für die Metaphysik der Erscheinungen liegen sie im Wissen, für die Metaphysik des Übersinnlichen und seiner Beziehungen zur Erfahrungswelt liegen sie in ,vernunftnotwendigen“ Glauben und im veruunftnotwendigen „Betrachten“. Nicht jedes Glauben oder jedes Betrachten hat dies metaphysische Recht, sondern nur das notwendige und allgemeingiltige, das v̀ernïnftige. Dies aber, das allein bcrechtigte, ans der Fïlle der individuellen und historischen Anspriiche herauszanschälen, bleibt auch bei Kant die Sache der Philosophie, der „wissenschaftlichen" Klärung; - ja, es ist ihre vornehmste Aufgabe. 
Damit steheu wir an den Punkte, wo die von Kant gegebeue Form des líritizisnus über sich selbst hinausweist. Das "Wissen", das er forträumen musste, um dem Glauben Plať zu machen, die "Wissenschaft", deren Anrecht an die Metaphysik in der Kritik der reinen Vernunft gewogen und zn leicht gefunden wird, - sie umspannen nicht den ganzen Unfang der theoretischen Erkenntnisarbeit. Kants Begriff der "Wissenschaft" ist - historisch sehr begreiflich - eingeengt auf den methodischen Charakter der theoretischen Naturforschung, bestimmt durch das Newtonsche Prinzip. Das kommt am deutlichsten und schroffsten bekanntlich in den „Metaphysischen Anfangsgründen der Naturwissenschaft" zu Tage, wo es heisst, dass „in jeder besonderen Naturlehre nur so viel eigentliche Wissenschaft angetroffen werden könne, als darin Mathematik anzutreffen ist", und wo deshalb Chemie und Psychologie von der "eigentlichen“ Wissenschaft ausgeschlossen werden. Aber auf denselben Begriff der "Wissenschaft" sind die Kritik und die Prolegomenen gestimmt: es ist lediglich der der Gesetzeswissenschaft.

Heutzutage ist dieser Begriff der Wissenschaft zu eng. Auf die Chemie fände Kant ihn jetzt vielleicht anwendbar, auf die Psychologie - trotz aller psychophysischen Gesetze - im Ganzen wohl kaum: und doch zählen wir auch sie zu den eigentlichen Wissenschaften. In noch ganz anderem Sinne aber gilt das von den historischen Disciplinen, die von Kant erst recht aus der Sphäre der Wissenschaft ausgeschlossen werden. Wir können ihm daraus keinen Vorwurf machen: denn bis zu seiner Zeit gab es in der That wesentlich nur eine Kunst der Geschichtsschreibung und grosse Künstler darin; aber die Geschichte zählte eben zu den belles lettres, sie war noch keine Wissenschaft. Dazu ist sie erst nach Kant geworden. Es gehört zu den eigentümlichsten Erscheinungen im geistigen Leben des neunzehnten Jahrhuuderts, dass neben der imposanten, namentlich nach aussen eindrucksvollen Entfaltung der Naturwissenschaft als ein stillerer, aber stetiger und zielsicherer Vorgang die Erhebung der Historie „zum Range einer Wissenschaft" einhergegangen ist. Wir haben jetzt die Geschichte als Wissenschaft, die Kant noch nicht gekannt hat. Und das ist nicht etwa daher gekommen, dass ein paar universalistische Methodologen und ein paar theoretisierende Historiker - niemals die grossen - gelegentlich das Verlangen gestellt haben, auch auf geschichtliche Vorgänge das Prinzip induktiver Aufsuchung 
von "Gesetzen" anzuwenden. Nein, diese Scientifikation der Historie verdanken wir einzig und allein dem kritischen Geiste, der sich von der phantasievollen Betrachtung der Vergangenheit her allmählich und mühsam zu streng methodischer Forschung erzogen und die dazu erforderlichen Verfahrungsweisen und Hilfsmittel mit vorsichtiger Anpassung an die eigenartige Natur der Gegenstände bis in das Einzelne hinein systematisch ausgebildet hat. Das schliesst nicht aus, dass in den abschliessenden Gesamtdarstellungen des wissenschaftlich Erworbenen und Gesicherten die künstlerische Genialität des grossen Forschers ihr Recht bebehält: gilt doch dasselbe auch für die überschauenden Leistungen des grossen Naturforschers und für den Abschluss alles Wissens in dem Sinue, wie es Schiller in den „Künstlern“ als höchstes Ziel geschildert hat.

Diese grosse neue Thatsache der Existenz einer historischen Wissenschaft verlangt nun von der kritischen Philosophie in erster Linie eine Erweiterung des Kantischen Begriffs vom Wissen: die Historie fordert neben der Naturforschung ihr Recht in der theoretischen Lehre. Auch ihr Wesen und ihr Erkenntniswert will, ihrer wirklichen Arbeit gemäss, verstanden und beurteilt werden. Damit verschieben sich Inhalt und Form der Wissenschaftslehre um ein beträchtliches gegenüber der Behandlung, die sie von Kant erfahren hat und unter den Voraussetzingen seiner Zeit erfahren musste. In der reinen Logik und in der Methodologie kann man schon seit langem, seit Lotze und Sigwart, die prinzipielle Berücksichtigung der Formen und Aufgaben des historischen Denkens neben dem naturwissenschaftlichen beobachten: in der Erkenntnistheorie ringt das gleiche Bestreben mit steigendem Erfolge nach Anerkennung.

Dabei aber stossen wir von Neuem, wenn auch in veränderten Begriffsformen, auf denselben Gegensatz, den Kant für die philosophische Weltanschauung in seiner Weise zwischen dem Wissen einerseits und dem vernunftnotwendigen Glauben und Betrachten andererseits gemacht hat. Wir finden die eine Art des wissenschaftlichen Denkens, die der Naturforschung, durchgängig und wesentlich durch das Bedürfnis bestimmt, aus den Thatsachen der Erfahrung dasjenige herauszuheben, was im Sein und Geschehen sich immer gleich bleibt: an der logischen Funktion des Gattungsbegriffs entwickelt sich die Forschung nach dem, was im Wechsel der Thatsachen beharrt einerseits als bleibendes Sein, anderseits 
als stetige Reihenfolge der Erreignisse - nach deu Snbstanzen und den Gesetzen ihrer Thätigkeit. Das . „Herauspräparieren“ dieser Ordnung aus dem Gewirr der Eindrücke gilt mit Recht als bewunderungswürdige Leistung des wissenschaftlichen Intellekts : aber eben damit ist schon gesagt, dass das so gewonnene Bild des Wirklichen nur einen Ausschnitt aus der "nnübersehbaren Mannigfaltigkeit" des Thatsächlichen bedeuten kann. Wenn dies Bild auch - um die Terminologie von Heinrich Hertz anzuwendeu - so adaequat wie möglich ist, so giebt es doch gerade dann nur diejenigen Momente der Wirklichkeit wieder, welche sich zil der Subsumtion unter die Gattungsbegriffe des Seins und des Geschehens eignen. Die lebendige Wirklichkeit des Einzelnen geht in diese allgemeinen Begriffe nicht ein: sie lassen sich darauf anwenden, aber sie erschöpfen sie nicht. Die so gewonnene "Erkenutnis" ist also nach der Auswahl, die unter den Thatsachen getroffen wird, und nach der Beziehung, die zwischen ihnen gesucht wird, eine Konstruktion der Vernunft, die ihre eigene logische (und mathematische) Gesetzmässigkeit in den Thatsachen entdeckt und daraus herauspräpariert hat. Die "Natur" als Objekt der Wissenschaft ist ein Kosmos, dessen Zusammenhang wir nur aus den Formen unserer Vernunft in Anschauungen und Begriffen vorzustellen vermögen: - genau wie es Kant gelehrt hat.

Aber ganz dasselbe gilt auch für die Geschichte -- mutatis mutandis. Auch der Historiker geht nicht daranf aus, das einzelne zeitliche Sein und Geschehen in seiner ganzen individuellen Mannigfaltigkeit $\mathrm{zu}$ beschreiben; er denkt um so weniger daran, als er das garnicht auszuführen vermöchte. Auch er trifft vielmehr aus der unendlichen Masse des geschichtlich Gegebenen eine Auswahl, welche keineswegs nur durch das Schicksal der Überlieferung, sondern vielmehr wesentlich durch das Interesse bestimmt ist, das die einzelnen Momente der Vergangenheit zu erwecken geeignet sind. Unsäglich vieles "geschieht", was niemals zur „Geschichte“ gehören wird. Das Interesse aber, das die Überlieferung wie die Auswahl des Historikers leitet, hängt in diesem Falle an den Wertbestimmungen des Menschenlebens; nur das ist historische Thatsache, was irgendwie für die Frinnerung der Gattung, für ihre wertbestimmte Selbsterkenntnis bedeutsam werden kann. Ebenso aber sind die Beziehungen, in die der Historiker die Thatsachen zu bringen hat, wesentlich durch dasselbe Interesse bestimmt: or sucht nicht Gattungsbegriffe, sondern Gestalten und 
Gestaltenkomplexe, die durch solche Wertbeziehungen bedingt sind. Nur als Mittel im Verständnis solcher Zusammenhänge benutzt auch die historische Forschung das Wissen von generellen Verhältnissen, das sie zum Teil den Gesetzeswissenschaften entlehnen kann, zum andern Teil aber selbst erst zu diesen Zwecken gewinnen muss. Auch die „Geschichte" also als Objekt der Wissenschaft ist ein geordneter Zusammenhang, den wir uns auf dem Grundriss allgemeingiltiger und notwendiger Vernunftinteressen aus dem Gegebenen herauszupräparieren vermögen: denn nur dadurch unterscheidet sich dabei die Wissenschaft von der individuellen Erzählung, dass sie an Stelle der persönlichen Interessen des Einzelnen die allgemein und notwendig geltenden Werte zum Prinzip der Auswahl und der Beziehung zwischen den Thatsachen macht.

Diese Erweiterung der erkenntnistheoretischen Untersuchung von den naturwissenschaftlichen auf die historischen Disciplinen, wie sie am besten von Rickert entwickelt und formuliert worden ist, führt nun unmittelbar darauf, für das "systematische Geschäft" der kritischen Philosophie die Allgemeinheit und Notwendigkeit der Werte, denen die Geschichte den Charakter als Wissenschaft verdankt, als das vollkommen ebenbürtige und parallele Problem zu der Apriorität der intellektuellen Formen erscheinen zu lassen, auf denen sich die Naturwissenschaft aufbaut. Die „Kritik der historischen Vernunft" leitet mit sachlicher Notwendigkeit und Selbstverständlichkeit zu den Aufgaben der Ethik, Ästhetik und Religionsphilosophie hinüber: sie leistet als Bindeglied dasselbe, was Kant durch die Konstruktion der transscendentalen Dialektik und durch die Beziehungen zwischen Ideen und Postulaten erreichen wollte. Sie zeigt aber dabei, dass nicht nur für eine sog. Metaphysik des Übersinnlichen, sondern schon für die unerlässliche Grundlage der historischen Wissenschaften die notwendige und allgemeine Geltung der Werte erforderlich ist.

Daher wird die Begründung dessen, was Kant den vernunftnotwendigen Glauben der praktischen Vernunft und die vernunftnotwendige Betrachtung der reflektierenden Urteilskraft genannt hat, d. h. die philosophische Theorie der Werte zum Mittelpunkt der Aufgaben, die der Fortentwickelung und systematischen Ausbildung des Kritizismus aus der gegenwärtigen Lage der Wissenschaften ebenso wie aus den allgemeinen Zuständen des geistigen Lebens erwachsen. Denn gerade in letzterer Hinsicht hat sich 
aus leidenscluaftlichen Impulsen, aus Gefühlen des Kraftüberschusses und der Expansionsbedürftigkeit, - wie aus den unausbleiblichen Kontraststimmungen decadenter Impotenz in dem allgemeinen Bewusstsein unserer T'age ein Individualismus und Relativismus entwickelt, der, nachdem er sich eine Zeit lang ausgetobt, bereits nach seiner Erlösung von sich selbst zu seufzen und zu drängen begonnen hat.

Solche Strömungen verdienen um so mehr Beachtung, als sie in gewissem Masse gegenüber den formalen Bestimmungen der kritischen Moralphilosophie in Rechte sind. Diese Bestimmungen sind eben in der That rein formal. Alle Werte, die Kants Ethik anerkennt, hängen an der Übereinstimmung des Besonderen mit allgemeinen Normen. In genauem Parallelismus zu dem Apriorismus seiner Erkenntniskritik gesteht Kant auch in Felde der Sittlichkeit die allgemeine und notwendige Geltung nur dem Generellen zu: dem Sittengesetz als einer Maxime, die als Naturgesetz gewollt werden kann. Und aus diesem Prinzip, das keinen andern Inhalt mehr haben soll als die Gesetzmässigkeit selbst, ist geflissentlich jede sachliche Bestimmung ausgeschlossen. Auch die Persönlichkeit, auf deren Autonomie so starkes Gewicht gelegt wird, empfängt ihre "Würde" nur von ihrer selbstgewollten Identifikation mit dem Vernunftgesetz, ihren Wert nur von der durch sie vollzogenen Verwirklichung des allgemeinen Pflichtgebots: ihre „Freiheit" besteht, dem positiven Begriffe nach, nur in der Fähigkeit, sich durch nichts anderes als durch das Gesetz bestimmen zu lassen. Diese Maximenmoral Kants ist schon in der ersten Zeit einem ästhetisch bewegten und stürmisch aufgeregten Geschlecht unbequem gewesen und unzulänglich erschienen: Jacobi, die Romantiker, auch Fichte häben diese Fesseln der Gesetzmässigkeit zu sprengen gesucht, und Schiller ist, so nahe er dem Prinzip der Pflichtmässigkeit bleiben wollte, doch dem Zauber der Unmittelbarkeit in der "sittlichen Natur" des Individuums nachgegangen.

Allein alle diese Versuche, die generellen Wertformen, auf die Kant die allgemeingiltigen Gründe des moralischen Urteils ausschliesslich zurückzufülhren für nötig befunden hatte, durch den Hinweis auf den gesicherten Bestand individueller Werterscheinungen $\mathbf{z u}$ ergänzen, - sie blieben entweder im Halben hangen oder sie stellten die Allgemeingiltigkeit und Notwendigkeit des Wertbewusstseins in Frage. Erst in dem grossen Sinne, womit 
Schleiermacher die Ethik lehrte, den ganzen Umfang des historischen Lebens zu umspannen und begrifflich $\mathrm{zu}$ bemeistern, fand sie auf diesem unermesslich erweiterten Arbeitsfelde auch das Verständnis der lebendigen Inhalte, welche als einmalige, individuelle Verknüpfungspunkte der generellen Norm-Beziehungen neben diesen selbst in ihrer Eigenart den Gegenstand allgemeiner und notwendiger Wertung ausmachen. In dieser Richtung allein kann die wesenhafte Entwickelung der kritischen Ethik gesucht werden: nur im unmittelbaren und methodischen Zusammenhange mit der Geschichtsphilosophie kann sie daran arbeiten, das formale Gerippe genereller Maximen mit dem Fleisch und Blut lebendiger Wertinhalte zu umkleiden. So vermag sie auch den gesättigten Reichtum der Hegelschen Lehre vom objektiven Geiste in sich aufzunehmen und die Verwirklichung der "Ideale" als das Wesen alles historischen Geschehens zu verstehen. Eben dadurch wird sie, zu einer Philosophie der Gesellschaft, zu einer kritischen Theorie des Gattungslebens: denn der Eigenwert der individuellen Gebilde der Geschichte besteht in ihrer eigenartigen, nur an ihnen in dieser Weise möglichen Beziehung zum Ganzen, zu der durch die Jahrtausende hin auseinandergelegten Entfaltung der Humanität. Darauf allein beruht die allgemeine und notwendige Geltung ihres Wertes. Sie zum Bewusstsein zu bringen und den Rechtsgrund der historischen Wissenschaft darin blosszulegen, ist die wesentliche Aufgabe der Ethik als allgemeiner kritischer Werttheorie und zugleich der unentbehrliche Ertrag, den sie für die philosophische Weltanschauung zu liefern berufen ist.

Denn nichts anderes kann doch schliesslich die Aufgabe der philosophischen Weltanschauungslehre - sagen wir doch ruhig der Metaphysik - sein, als uns darüber zu verständigen, welches Recht wir haben, dem objektiven Weltbilde, das uns die Wissenschaften als das notwendige und allgemeingiltige Denken der Menschheit darbieten, die Kraft zur Erfassung der Realität, der absoluten Wirklichkeit zuzutrauen. Das ist die Frage der Erkenntnistheorie und - der "Metaphysik". Denn von dem Verhältnis des objektiven Denkens zum Realen kann man nicht reden, ohne vom Realen zu reden - selbst wenn man es das unerkennbare Ding-an-sich nennt. Die "Metaphysik des Wissens" - so hat Kant mit Hume die kritische Philosophie genannt - ist auch eine Metaphysik der Dinge. Aber freilich keine solche, welche aus ontologischen Prinzipien ein eigenes Begriffssystem in der „freien 
Luft der Einbildungskraft" ausführt, sondern eine solche, welche aus den Argumenten der Sonderwissenschaften, die sie in ihrem Bestande als objektives, allgemein und notwendig geltendes Denken unangetastet und unerschüttert bestehen lässt, die kritische Frage entscheidet, in welchem Sinne sie selbst eine „Erkenntnis der Wirklichkeit" zu sein beanspruchen dürfen. Das ist das Fundament, welches Kants Kritiken ein für alle mal für alle weitere Philosophie, für „eine jede künftige Metaphysik, die als Wissenschaft wird auftreten können,“ in unverrückbarer Sicherheit gelegt haben.

In diesem Sinne steht die kritische Philosophie, wenn es sich um die letzten Fragen handelt, vor dem grossen Gegensatze der Gesetzeswissenschaften und der Wertwissenschaften, - der Naturforschung und der Historie. Jede von ihnen enthält in der Auswahl und in den Beziehungen der Thatsachen ein Produkt des objektiven Denkens, das in seiner gesamten Struktur durch die verschiedenen $\mathrm{Zwecke}$ dieses allgemeingiltigen und notwendigen Denkens bestimmt ist: auf der einen Seite eine Ordnung von Gattungsbegriffen, auf der andern ein System von Werten. Jeder einzelne Bestandteil unseres Erfahrungsmaterials kann unter jede der beiden wissenschaftlichen Bearbeitungsweisen fallen, und ein grosser Teil der besonderen Disciplinen wendet sie in einer Verbindung an, über die man sich durchaus nicht immer und überall prinzipiell klar ist und bei der einzelnen praktischen Forschungsarbeit auch nicht klar zu sein braucht. Diese Verhältnisse festzustellen ist Sache der Methodologie, deren Geschäft ja kein anderes ist und sein kann, als die Wissenschaften über den logischen Sinn und Wert dessen zu verständigen, was sie in unmittelbarer Bewältigung ihrer Aufgaben eigentlich thun. Aber wenn so die beiden Grundformen der Auswahl und Beziehung von Thatsachen, am Einzelnen angewendet, sehr verschiedene und scheinbar weit auseinander liegende Systeme der Erkenntnis des Wirklichen ergeben, so erwächst für die Philosophie eben damit durch die Anwendung derselben Dualität der Behandlungsweisen auf das Ganze die höchste ihrer Fragen: wie verhält sich das Reich der Gesetze zu dem Reich der Werte?

Das ist, wie man sieht, genau das Problem der Kritik der Urteilskraft, das in den Kantischen Formeln auf die Vereinbarkeit von Natur und Zweckmässigkeit, von Notwendigkeit und Freiheit hinauslief. Unter den Denkern des neunzehnten Jahrhunderts hat 
dies Problem keinor so klar gesehen und so deutlich formuliert wie Lotze: seine ganze Lehre des "teleologischen Idealismus" läuft - genau wiederum im Sinne der Kritik der Urteilskraft darauf hinaus, in der Gesamtheit der Gesetze das System der Formen zu sehen, durch welche sich eine inhaltliche Welt der Werte verwirklicht. Das beruht - wiederum wie in Kants "Metaphysik" -- auf der tiefen Einsicht, dass aus den allgemeinen Formen des Geschehens, den ewig sich gloich bleibenden Gesetzen, niemals der lebendige Inhalt herstammen oder begriffen werden kaun, den unser Wertbewusstsein in der Wirklichkeit sucht und findet.

Aber der übergreifende Begriff, der in dieser Weise das Reich der Gesetze mit dem der Werte verbindet, ist der der Verwirklichung, die höchste Kategorie der Weltbetrachtung ist das Verhältnis des Mittels zum Zweck: es ist das Prinzip der Entwickelung. Seine beherrschende Stellung tritt in der Kritik der Urteilskraft deutlich genug hervor: es ist das heuristische Prinzip fiir die vernunftnotwendige Betrachtung des "Lebens": es ist der leitende Gedanke in jenen gewaltigen Paragraphen am Ende des Werkes, wo alle Linien der Kantischen Philosophie zusammenlaufen, wo der zweckvolle Zusammenhang der Natur als der gesetzmässigen Ordnung der Erfahrungsinhalte und der wertbestimmte Ablauf der ganzen Menschengeschichte mit einander als die fortschreitende Verwirklichung des Reiches Gottes auf Erden „vernunftnotwendig“ betrachtet werden.

An dieser Stelle setzt mit Schelling in erster Linie Hegels Gedankenarbeit in der Weiterbildung des Kritizismus ein: hier gelangt der historische Charakter der neuen Weltanschaung zum entscheidenden Durchbruch. Danit freilich kommt der Kantischen Erkenntnislehre gegenüber eins der schwierigsten Probleme zum Vorschein, das hier nur angedeutet werden unag. Wenn die Entwicklnng als das reale Wesen des Lebens und des Universums vernunftnotwendig betrachtet werden soll, - wie ist damit die Phaenomenalität der Zeit vereinbar? Wenn die Werte in der Verwirklichung begriffen, nur in ihr zu verstehen sind, so muss das Geschehen, das ohne Zeit nicht denkbar ist, eine wesentliche Bestinmung des Wirklichen' selber sein, so darf es nicht bloss als Form der Anschaumng gelten. So stellt die Lehre von der Entwicklung - das liesse sich auch an Kants Ethik und Religionsphilosophie anfweisen - die parallele Behandlung voll Kantstudies $1 \mathbf{X}$. 
Raum und Zeit, wie sie die transscendentale Ästhetik eingefiihrt hat, unausweichlich in Frage.

Die Bedeutung des Prinzips der Entwicklung für die moderne Naturwissenschaft ist allgemein bekannt: weniger verbreitet und anerkannt ist die Einsicht, dass es ein Wertprinzip ist. Und doch ist das kaum deutlicher $\mathrm{zu}$ machen, als es schon Kant in der Kritik der Urteilskraft eben da gethan hat, wo er die bedeutsamsten Anwendungen dieses Prinzips auf die Gesamtauffassung des organischen Lebens und seines geschichtlichen Zusammenhanges voraussehend in grossen Linien gezeichnet hat. Wer sich darüber keine Gedanken macht, der mag wohl naiv von höheren und niederen Formen, von der Herausbildung der ersteren aus den letzter $\in \mathrm{n}$, von uormalen und abnormen Bildungen u. s. w. reden: wollte er sich darauf besinnen, nach welchen Kriterien und mit welchem Rechte er solche ihm geläufigen Unterscheidungen anwendet, so würde er auf die Wertbestimmungen stossen, deren er beim Verständnis des Lebens nun und nimmermehr entraten kann. Gerade da, wo man gemeint hat, nun endlich das Wunder aus der Welt des Mechanismus verjagt zu haben, dem Geheimnis der Zweckmässigkeit auf der Spur zu sein, -- gerade da hat man dem Gast aus der Welt der Werte das Bürgerrecht im Reiche der Gesetzeswissenschaften gegeben.

In der fruchtbaren Anwendung dieses Prinzips auf die Erkenntnis der Körperwelt besteht offenbar der wesentliche Beitrag, den die Naturforschung des neunzehnten Jahrhunderts für die philosophische Weltanschauung geliefert hat. Mit der Formulierung des Prinzips von der Erhaltung der Energie hat sie fast gleichzeitig die allgemein und notwendig giltige Grundvoraussetzung aller Gesetzeswissenschaft auf den glïcklichsten Ausdruck gebracht. Das Prinzip, dass es in der Welt nichts Neues geben könne, dass alles Sein und Geschehen jedes Moments nicht mehr und micht weniger enthalten könne als das des vorhergehenden, ist darin auf die universell brauchbarste Weise niedergelegt. Aber dieser Vorstellung von der quantitativ gleichbedeuteuden Reihe der Umwandlungen fügt nun auch im Umkreis des körperlichen Geschehens der Entwicklungsgedanke das Verständnis hinzu, dass der qualitative Inhalt djeser Umwandlungen nach den Werten zu beurteilen ist, die sich darin verwirklichen. Diese gegenseitige Ergänzung der beiden grossen Prinzipien der Naturforschung scheint das Thenla der heutigen Naturphilosophie zu werden; welche in Energetik und 
Neovitalismus den lang geschmähten Namen wieder zu Ehren bringt. Je mehr sie mit der Erneuerung einer dynamischen Auffassung von unorganischer und organischer Welt zu dem Bestreben zurückkehrt, hinter den quantitativ bestimmten Formen des Geschehens den Inhalt zu suchen, der sich darin verwirklicht, um so mehr nähert sie sich auf ihrem Gebiete der von Lotze formulierten Aufgabe, den Weltlauf zu verstehen und ihn nicht bloss zu berechnen.

So drängt Alles darauf hị, dass die kritische Philosophie, wenn sie die Lebenskraft, die sie ein Jahrhundertlang bewahrt hat, auch in der Bewältigung der intellektuellen Bedürfnisse der Gegenwart bewähren soll, sich fähig erweisen muss, mit ihrem Begriffsystem eine Weltanschauung zu tragen, welche den geistigen Wertinhalt der Wirklichkeit in sicherem Bewusstsein zu erfassën vermag. Sie hat dazu das Recht und den Beruf, weil sie, deu Kantischen Grundlagen gemäss, die Gründe allgemein giltiger und notwendiger Überzeugungen in dem ganzeu Umfange menschlicher Kulturthätigkeit, im sittlichen und geschichtlichen Leben, in Kunst und Religion ebenso wie in der Wissenschaft zu suchen angewiesen ist. Und diese Aufgabe zu erfüllen, dazu stehen die Zeichen der Zeit nicht ungünstig. Schon erleben wir - fast von Tage zu Tage - einen rapiden Niedergang der naturalistischen Weltanschauung, die nur noch gelegentlich einmal von einem der Alten, die nichts mehr gelernt haben, mit glücklicher Ahnungslosigkeit aufgetischt wird. Unsere Jugend, die die Macht des historischen Lebens in sich fühlt, - sie brennt darauf, ihre gährenden Wertgefühle in klare Begriffe umgesetzt zu finden. Es ist alle Hoffnung, dass der gute Kampf um einen geistigen Lebensinhalt, wie ihn Eucken mit edler Leidenschaft kämpft, zuın Siege führe.

Wenn wir die Werte des geistigen Lebens, das das geschichtliche ist, für den letzten Inhalt aller Wirklichkeit ansehen, dessen Verwirklichung herbeizuführen auch den Sinn alles natürlichen Geschehens mit dem ganzen Apparat seiner gesetzmässigen Kausalität bilde, so wird wohl erwidert: das sei und bleibe Anthropologismus und Anthropomorphismus. Ob man denn immer noch nicht gelernt habe, dass unser gesamtes Geschlecht mit seinem Leid und Lust, mit seinem Meinen, Wüuschen und Wollen in einem entlegnen Winkel des Univiersums eine beschränkte, auf kurze. Frist gespannte Existenz abspielt! Woher das Recht, das 
was uns bewegt, als Werte zu betrachten, die in den letzten Tiefen aller Wirklichkeit wurzeln sollen?

Das klingt sehr einsichtig und ist doch sehr kurzsichtig. Gewiss, der Hegelsche "Weltgeist" als der Inbegriff der Kategorion der Wirklichkeit, er ist in Wahrheit der Menschengeist in der historischen Entwickelung seiner inneren Wertbestimmungell. Aber gehört nicht $\mathrm{zu}$ eben diesen Errungenschaften des historischen Geistes auch jenes Wissen von den gesetzmässigen Zusanmenhängen eines in Raum und Zeit unendlich ausgebreiteten Weltalls, wozu sich Schritt für Schritt die simnlich beschränkte Vorstellung unserer physischen Existenz erweitert und umgebildet hat? Verdanken wir dieser unserer eigenen geistigen Arbeit den Einblick in die physische Ordnung der Dinge, der wir anzugehören überzeugt sind, fühlen wir uns damit in einen Zusammenhang gerückt, der weit über uns selbst hinausreicht, so erhebt uns das geschichtliche Leben in eine geistige Ordnung, die an allen Ecken und Enden über sich selbst hinaus, über alles menschliche Drängen und Treiben in eine höhere weltumspannende Wirklichkeit weist. Wenn irgend eine, so ist es die Aufgabe der Religionsphilosophie, dies zur deutlichen Besinnung zu bringen. Wir kennen die Werte der geistigen Wirklichkeit nicht anders als durch unsere Geschichte, in der sie sich zur Geltung herausgerungen haven, gerade wie wir die Gesetze des physischen Daseins nicht anders kennen, als durch die Formen unseres Intellekts, die wir darin walten gefunden haben: und eben deshalb haben wir das Recht überzeugt zu sein, dass auch die Werte des geistigen Lebens, zu denen unsere geschichtliche Entwickelung aufringt, ebenso lebendige Wirklichkeit sind wie die Sonnensysteme. Mit dem Reiche der Gesetze, die wir denken, und mit dem Reiche der Werte, die wir erleben, wissen wir uns gleichermassen in den grossen Ordnungen eines Weltzusammenhanges, die mit gleichem Rechte unsere Ehrfurcht verlangen: „der bestirnte Himmel über mir und das Sittengesetz in mir." 